\title{
PRE-TRIGGERED FLASH TUBES FOR PARTICLE DETECTION*
}

\author{
J. H. $\mathrm{COBB}^{\dagger}$ \\ Brookhaven National Laboratory, Lipion N.Y., U.S.A. \\ D. J. MILLER \\ Lniversity College London, London, England \\ and \\ E. ROSSO \\ CERN, Geneva, Switzerland
}

Received 9 January 1978

A new form of flash tube has been investigated as a particle detector, with special interest in its performance at liquid nitrogen tempcrature. The results indicate that the tubes count efficiently and with sufficiently good time resolution to be able to assist in resolving independent events in bubble-chamber neutrino pictures.

\section{Introduction}

It is difficult to find a particle detector which gives good time resolution, covers a large area with some spatial resolution and which will work reliably when mounted inside a bubble chamber. Scintillation counters require too large an area of light-guide to be brought through the walls of the chamber. Multiwire proportional chambers cannot operate at cryogenic temperatures. Solid argon ionisation chambers') give such a weak signal that their pre-amplifiers would need to be mounted close to the detector in a harsh and inaccessible environment. In a search for some suitable Internal Fast Detector (I.F.D.) to be placed inside the large CERN bubble chamber BEBC, a new mode of operation of flash tubes has been investigat$\mathrm{ed}^{2,3}$ ). The results, reported here, suggest that a simple and robust flash-tube system can be developed which will provide important extra information in the analysis of neutrino pictures from BEBC.

The novelty in these investigations was to operate flash tubes with a square high-tension pulse of a few milliseconds duration. Each tube was sensitive to the first charged particle which passed through it after the start of the pulse. This is in contrast to normal flash tubes which are pulsed af-

* Work done at CERN. A fuller account of this work is available as a CERN report.

+ Present address: c/o E. P. Division, CERN, Geneva, Switzerland. ter the particles have already passed through and been detected by a scintillator $\left(\operatorname{trigger}^{3}\right)$.

\section{Apparatus}

The square ht pulse was generated using a hydrogen-thyratron pulser connected to a high voltage generator. Pulses of between 3 and $17 \mathrm{kV}$ were delivered to the flash-tube electrodes, with a duration that could be varied from a few hundred microseconds to a few milliseconds. The rise time was about $10 \mu \mathrm{s}$ and the fall-off time, after the flat-top of the pulse, about $1 \mathrm{~ms}$.

Two sets of flash tubes were used for these studies. The first set of eight tubes were of sandwich construction, each bank of tubes being made by sticking transparent Lexan ${ }^{4}$ ) sheet, $1 \mathrm{~mm}$ thick, over the two faces of a $10 \mathrm{~mm}$ thick transparent Lexan spacer into which four parallel slots had been milled, each $15 \mathrm{~mm}$ wide by $313 \mathrm{~mm}$ long. Gas was supplied to the tubes in series and a brass probe $1 \mathrm{~mm}$ in diameter projected $8 \mathrm{~mm}$ into the end of each tube. Aluminium electrodes were clamped around the two banks of tubes, with the ht electrode in the middle and the two ground electrodes on the outside (sce fig. 1). The ht electrode stopped about $20 \mathrm{~mm}$ short of the ends of the tubes, so that the tips of the probes lay outside the high field region. The probes were connected to NIM discriminators by $30 \mathrm{~m}$ of $50 \Omega$ signal cables, with the sheaths connected to the ground electrodes. Holes in the electrodes allowed $\beta$-rays from a ${ }^{106} \mathrm{Ru}$ source to penetrate into any one of the tubes. 

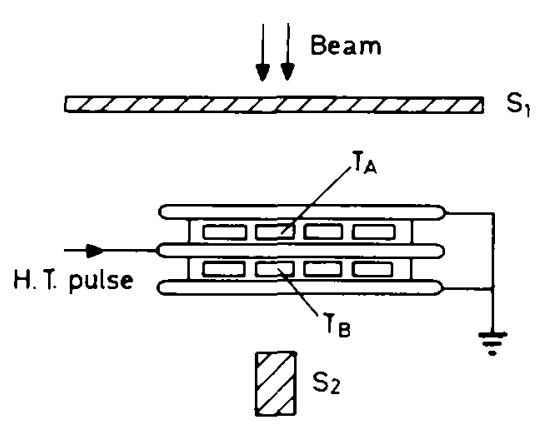

a) Transparent tubes
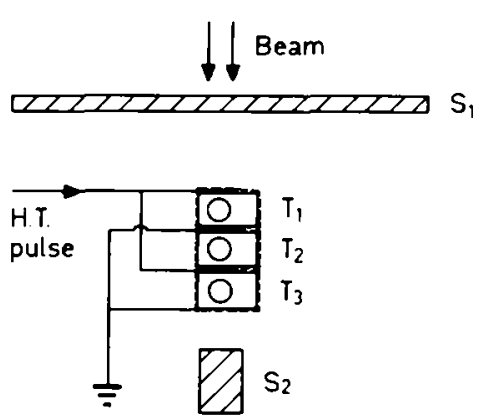

b) Wrapped tubes

Fig. 1. The layoul of the two sets of llash tubes in the beam from the CERN P.S. $S_{1}$ and $S_{2}$ are scintillation eounters. $T_{A}$ and $\mathrm{T}_{B}$ are the pair of tubes from the transparent set which were counted in coincidence.

After some experience with these "transparent tubes" a second set of three "wrapped tubes" was made. These were separate single tubes, each with $8 \mathrm{~mm}$ inside diameter by $300 \mathrm{~mm}$ long, made by boring through a transparent Lexan rod which was milled down to a rectangular external section $11 \mathrm{~mm} \times 16 \mathrm{~mm}$. Lexan plugs were stuck into the ends and gas channels were provided so that the tubes could be connected in parallel. A brass probe in each tube reached to the edge of the high-field region. Copper foil electrodes were placed on both $16 \mathrm{~mm}$ faces of two of the tubes and stuck down by folding a piece of black PVC tape over the tube and the foil (see fig. 1). The tubes were bound together so that no light could get into them from outside or pass from one tube to another. The four foil clectrodes were connected alternately to the ht supply and to ground and the probes were connected, as before, to $50 \Omega$ signal cables. Since the tube walls were thin, tests with the $\beta$-source could be made very simply by placing it in contact with the wrapping of one of the three tubes.

The gas in the tubes was Henogal $(75 \% \mathrm{Ne}-25 \% \mathrm{He}$ mixture) at slightly above atmospheric pressurc. For certain tests, as mentioned below, small amounts of nitrogen or hydrogen were added.

\section{Tests with the transparent tubes}

At room temperature, with the $\beta$ source, an investigation was made of the factors which caused variations in the performance of the tubes. Three aspects of performance were looked for; noise counts without source, crosstalk between adjacent tubes and genuine counts due to $\beta$-rays. The noise counts were found to increase with the rate of gas flow, with the ht pulse voltage and with the am- bient lighting intensity. Noise counts were also more probable if the tube had just flashed on a previous pulse. Crosstalk was caused by visible light from one tube triggering a flash in adjacent tubes. The crosstalk was worst in the same conditions which gave rise to high noise rates; i.e. with a high gas flow $(\sim 11 / \mathrm{min})$ and a high voltage $\gtrsim 7 \mathrm{kV}$. With a lower gas flow $(\sim 0.11 / \mathrm{min})$ it was possible to reduce noise and crosstalk to a low level, and to see clear $\beta$-ray pulses coming from the probe in the tube over which the source had been placed. In these conditions there was a clear counting plateau over the range of ht voltages from 4 to $7 \mathrm{kV}$. The height of the pulses from the probes was proportional to the termination resistance of the signal cables. With $6.5 \mathrm{kV}$ on the ht supply the pulse heights were $100-200 \mathrm{mV}$ into $50 \Omega, 2-3 \mathrm{~V}$ into $1000 \Omega$ and well over $40 \mathrm{~V}$ into the high impedance oscilloscope input. All counting with the transparent tubes was done using a discriminator with a $50 \Omega$ input impedance and a bias of between 40 and $70 \mathrm{mV}$. The rise-time of the pulses was $\sim 200 \mathrm{~ns}$ as observed at the end of $\sim 30 \mathrm{~m}$ of cable.

A special test was made with the probes from two adjacent tubes connected to one coaxial signal cable. When one tube flashed the other one did not brcak down, though a normal pulsc was observed on the cable. Occasionally a second pulse would be seen on the signal cable due to the two tubes flashing separately at different times. Only in this situation with two tubes connected in parallel, did two signal pulses appear on the same cable during one ht pulse. Otherwise the tubes always were dead once they had fired, until the high voltage was removed. But within a few hundred microseconds of the removal of the high 
voltage, tubes which had flashed were observed to flash for a second time, and also to give a simultaneous signal pulse of the opposite sense to the primary signal pulse. These "return pulses" were interpreted as the discharge of ionisation from the walls of the tubes which had been held there after the plasma of the primary discharge had quenched.

With a particle beam from the CERN proton synchrotron passing through the tubes, as shown in fig. 1, a number of coincidence combinations were set up between the tubes and the scintillators $S_{1}$ and $S_{2}$. At low gas flow, with an ht voltage of $8.5 \mathrm{kV}$, tubes $\mathrm{T}_{A}$ and $\mathrm{T}_{B}$ counted in coincidence with better than $90 \%$ efficiency. Due to crosstalk between tubes in the same bank, more detailed studies of efficiency were delayed until the wrapped tubes were available (sec below).

The advantage of Lexan over plexiglass is that it can be cooled to cryogenic temperatures without becoming brittle. As a first test of the low temperature performance of flash tubes, avoiding the complications of working at liquid hydrogen temperature, the transparent tubes and their electrodes were immersed in liquid nitrogen in a glass Dewar. When the ht pulse was first applied to the tubes, after cooling down, they gave signals from the beam in the same way as when warm, but after a few hundred pulses they began to flash as soon as the ht was applied, without waiting for a particle to arrive. A number of unsuccessful techniques were used to try and suppress this earlypulsing; a reverse cleaning field, a rest of a few minutes from ht pulses, variations in the rate of glas now, the addition of nitrogen to the gas. Finally it was suggested ${ }^{5}$ ) that a small amount of hydrogen should be added to the Henogal flow. This immediately cured the early pulses and the tubes began to behave when cold in the same way as they had done warm. Further cold studies were carried out with the wrapped tubes.

\section{Tests with the wrapped tubes}

Because the probes in the wrapped tubes penetrated right to the edge of the glowing plasma the pulse-height into $50 \Omega$ was larger than before; 3 or $4 \mathrm{~V}$ with an applied ht of $6 \mathrm{kV}$. At higher ht voltages this gave rise to electronic crosstalk between the signal cables at first, but this was eliminated by careful screening and by adding a $9.1 \Omega$ resistor directly between each probe and the grounded sheath of its signal cable. The rise time of the sig- nal pulses was less than $100 \mathrm{~ns}$ and the falling edge had a decay time of about 200 ns.

After successful tests with the $\beta$-ray source, the tubes were placed in the beam (see fig. 1). Singles counts from scintillator $S_{1}$ and the tubes $T_{1}, T_{2}$ and $T_{3}$ were recorded, and coincidences were set up for $S_{1} \cdot S_{2}, T_{1} \cdot T_{3}, t_{1} \cdot T_{2} \cdot T_{3}$ and $S_{1} \cdot T_{1}$. To simimulate the dead-time of a flash tube, only the first $S_{1} \cdot S_{2}$ count was recorded within the counting gate which covered the flat-top of the ht pulse. The area of scintillator $\mathrm{S}_{2}$ was approximately the same as that of a flash tube so this combination provided a good normalisation for variations in beam intensity. The $S_{1} \cdot T_{1}$ coincidence was used to trigger a pulse-height analyser on which the distribution of delay times was recorded between the scintillator pulse and the flash tube pulse. The ratio of the $T_{1} \cdot T_{2} T_{3} / T_{1} \cdot T_{3}$ coincidences gave an estimate of the efficiency of tube $T_{2}$.

Fig. 2a shows the variation of some of these counts with ht voltage. The counting statistics are low because only one count could be accepted per cycle of the proton synchrotron. Nevertheless, very clear plateaux can be seen. The reduction of $S_{1} \cdot T_{1}$ coincidences, compared with $T_{2}$ singles, at the highest voltages is due to noise on tube $T_{1}$. The ratios $\mathrm{T}_{1} \cdot \mathrm{T}_{2} \cdot \mathrm{T}_{3} / \mathrm{T}_{1} \cdot \mathrm{T}_{3}$ and $\mathrm{S}_{1} \cdot \mathrm{T}_{1} / \mathrm{T}_{2}$ are slightly less than 1.0 due to the high rate of singles counts, coming from neutral and uncollimated particles in the test beam. These ratios varied according to the thickness of the equipment which upstream users were testing. When these background effects were allowed for, the efficiency of tube $\mathrm{T}_{2}$ was consistent with $100 \%$, with no sign of any noise or crosstalk from 6 to $16 \mathrm{kV}$.

For the cold tests it was necessary to put more material very close to the flash tubes: a copper gas-cooling coil, the glass Dewar and the liquid nitrogen. Preliminary checks with the warm tubes showed that such material increased the background singles rates even more, compared with the good bcam. Thus it is not surprising that, in fig. $2 b$, the $S_{1} \cdot T_{1}$ counts rise to a smaller fraction of the $T_{2}$ singles rate than in fig. 2a. Nevertheless, when background singles are unfolded from the points in fig. $2 b$, especially the high statistics points at $9 \mathrm{kV}$ and $10 \mathrm{kV}$, the efficiency of $T_{2}$ was again consistent with $100 \%$. Because of the crude gas system used, it was necessary to keep the Henogal flow at about $1 \mathrm{l} / \mathrm{min}$ in order to dilute the smallest controllable hydrogen flow, and the tubes were connected in a way which led $T_{1}$ to re- 


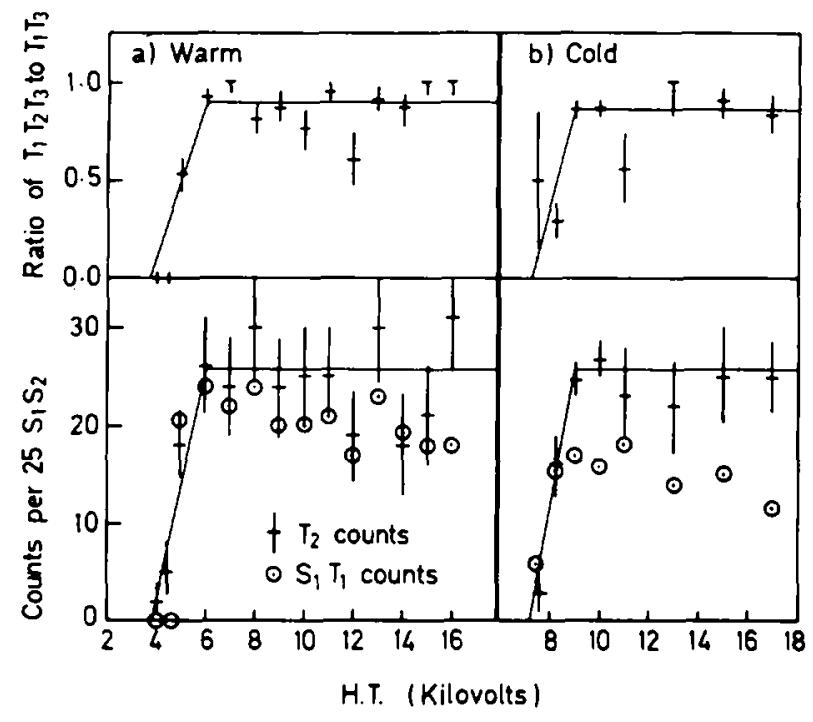

Fig. 2. Plateau curves for the wrapped tubes, (a) at room temperature, (b) in liquid nitrogen. The lower plots are normalised to 25 counts of the $S_{1} \cdot S_{2}$ coincidence with simulated deadtime (sec text). The straight lines are merely to guide the eye.

ceive a disproportionate share at high rates of flow. This accounts for the increase of noise on tube $T_{1}$ which can be seen in the drop of $S_{1} \cdot T_{1}$ coincidences at higher voltages. Tubes $T_{2}$ and $T_{3}$ were a great deal less noisy than $T_{1}$, as judged by their counting rates without beam, and fig. $2 \mathrm{~b}$ shows no sign of noise effects on $T_{2}$ even at $16 \mathrm{kV}$.

The time delay between pulses from scintillator $S_{1}$ and from tube $T_{1}$ was converted to a pulseheight and histogrammed on a pulse-height analyser. The time-jitter of the flash-tube signals was taken to be the range of delay times containing $90 \%$ of $S_{1} \cdot T_{1}$ coincidences. With the wrapped tubes warm the time-jitter was $80 \mathrm{~ns}$ at $10 \mathrm{kV}$ and $110 \mathrm{~ns}$ at $6 \mathrm{kV}$. With the wrapped tubes in liquid nitrogen the time-jitter was $420 \mathrm{~ns}$ at $10 \mathrm{kV}$ and $585 \mathrm{~ns}$ at $9 \mathrm{kV}$.

\section{Conclusions}

Flash tubes made from Lexan can be operated in a pre-triggered mode at a repetition rate compatible with bubble-chamber neutrino experiments. Their efficiency is high over a finite range of applied voltages, both a room temperature and at liquid nitrogen temperature. Pulses can be extracted on conducting probes which reach into the gas of the tubes. The pulses are large enough to drive a NIM discriminator without pre-amplification. They have a rise time of $\sim 100 \mathrm{~ns}$ and a time jitter of a few hundred ns.

Further tests are continuing to investigate the performance of pre-triggered flash tubes at liquid hydrogen temperature, to understand the quenching of early pulses by hydrogen, to look for other quenching techniques and to develop suitable large area modules which could be mounted on the inner wall of the BEBC bubble chamber at CERN.

I. Mannelli first suggested this form of flash tube and took part in the earlier tests at CERN ${ }^{2}$ ). We are grateful for advice and encouragement from M. Conversi and C. Peyrou; also for help from G. Trouillier, A. Rudge, T. Broome, M. Firth, J. Tischauser, M. Thevenon, H. Leutz, W. Willis and C. Fabian.

\section{References}

I) J. Cobb and D. J. Miller. Nucl. Instr. and Meth. 141 (1977) 433.

2) First evidence for successful operation in pre-triggered mode came from S. Pass and S. Fedida (University College London, teaching-laboratory report, unpublished) and I. Mannelli and E. Rosso (CERN internal note. unpublished).

3) For references on flash tubes in the conventional mode sce e.g. review by $\mathbf{M}$. Conversi and L. Federici, Flash chambers of plastic material, CERN report. Nucl. Instr. and Meth. 151 (1978) 93; work by J. H. Brear et al., e.g. Nucl. Instr. and Meth. 138 (1976) 457; or M. Conversi and G. Brosco, Ann. Rev. Nucl. Sci. 23 (1973) 75.

4) Lexan is the tradename for polycarbonate plastic, manufactured by the General Electric Company, U.S.A.

5) L'se of $\mathrm{H}_{2}$ was suggested independently by $\mathrm{M}$. Conversi and C. Peyrou. 